\title{
Synthesis of Novel O-Acylated-D-ribono-1,5-lactones and Structural Assignment Supported by Conventional NOESY-NMR and X-ray Analysis
}

\author{
Marcus M. Sá, ${ }^{*, a}$ Gustavo P. Silveira, ${ }^{a}$ Miguel S. B. Caro ${ }^{a}$ and Javier Ellena ${ }^{b}$ \\ ${ }^{a}$ Departamento de Química, Universidade Federal de Santa Catarina, 88040-900 Florianópolis-SC, Brazil \\ ${ }^{b}$ Instituto de Física de São Carlos, Universidade de São Paulo, CP 369, 13560-970 São Carlos-SP, Brazil
}

\begin{abstract}
Este trabalho apresenta um método simples para a caracterização estrutural de 3,4-O-benzilideno-D-ribono-1,5-lactonas e análogos, fundamentado em técnicas convencionais de RMN e experimentos de NOESY em solução. 2- $O$-Acil-3,4- $O$-benzilideno-D-ribono-1,5-lactonas foram preparadas em bons rendimentos a partir da acilação das lactonas de Zinner empregando cloretos de ácido sob condições básicas. A estrutura de 2-O-(4-nitrobenzoil)-3,4-O-benzilidenoD-ribono-1,5-lactona foi determinada por difração de raios-X e confirmou, de forma inequívoca, os dados espectroscópicos obtidos.
\end{abstract}

A practical method for the structural assignment of 3,4-O-benzylidene-D-ribono-1,5-lactones and analogues using conventional NMR techniques and NOESY measurements in solution is described. 2-O-Acyl-3,4-O-benzylidene-D-ribono-1,5-lactones were prepared in good yields by acylation of Zinner's lactone with acyl chlorides under mildly basic conditions. Structural determination of 2-O-(4-nitrobenzoyl)-3,4- $O$-benzylidene-D-ribono-1,5-lactone was achieved by single crystal $\mathrm{x}$-ray diffraction, which supports the results based on spectroscopic data.

Keywords: O-acyl-D-ribono-1,5-lactones, D-ribono-1,4-lactones, structural elucidation, sugar lactones, acylation

\section{Introduction}

Several carbohydrates are easily available from renewable resources ${ }^{1,2}$ and constitute a major class of building blocks for the synthesis of natural products and biologically active compounds. ${ }^{3-7}$ However, the synthesis of novel carbohydrate-based molecules and their unequivocal structural elucidation are usually difficult to accomplish by simple and predictable methods. ${ }^{8-10}$ The chemical complexity of carbohydrate derivatives, due to subtle differences in reactivity of the various hydroxyl groups and the possible formation of species co-existing in equilibrium under different reaction conditions, may lead to unexpected processes ranging from rearrangements to functional group migrations. ${ }^{11,12}$ In many cases, single crystal X-ray analysis is the only reliable method for correct structural and conformational assignments. ${ }^{13,14}$ Aldonolactones ${ }^{15}$ (modified sugars with the anomeric centre in its higher oxidation state) have been widely employed in synthesis as an alternative to simple carbohydrates. D-Ribono-1,4-lactone (D-ribono- $\gamma$-lactone, 1 ) is one of the most useful chiral precursors from this class, ${ }^{16-19}$ although the issues associated with product characterization due to unpredictable transformations are also present to a great extent. For instance, the product obtained from the reaction of D-ribono-1,4-lactone (1) with benzaldehyde in acidic medium, which was initially assigned as being 3,5-O-benzylidene-D-ribono-1,4-lactone (2), ${ }^{20,21}$ had the correct structure unequivocally established as 3,4-O-benzylidene-D-ribono-1,5-lactone (3) by single crystal X-ray diffraction analysis of its $\mathrm{O}$-acetyl derivative (Scheme 1). ${ }^{22}$ A complex rearrangement involving acetal migration and ring expansion sequences has been proposed for this transformation. ${ }^{10,14}$ Therefore, the scope of D-ribono-1,5-lactone 3 and analogues as chiral building blocks ${ }^{23-27}$ relies on the development of more convenient
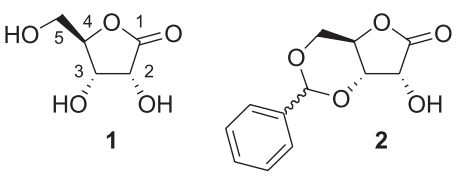

Scheme 1.

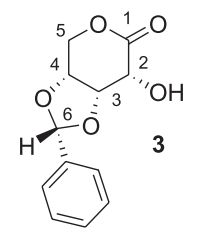


methods for structural assignment, particularly those based on more conventional NMR techniques. ${ }^{8,28-30}$

In our ongoing studies on synthetic transformations of nucleosides and carbohydrates as inhibitors of trypanosomal gGAPDH enzyme, ${ }^{31-33}$ the synthesis of selectively acylated acetals of D-ribono-1,5-lactone 3 was required for screening programs. Based on the above statements and bearing in mind that derivatives of $O$-benzylidene-D-ribono1,5-lactone 3 undergo acid-catalyzed deprotection with subsequent rearrangement to the corresponding D-ribono1,4-lactones, ${ }^{13}$ we anticipated that any further chemical transformation on $\mathbf{3}$ should not be merely assumed without proper spectroscopic support. ${ }^{34,35}$ In this paper we describe a simple and efficient method for structural assignment of benzylidene-D-ribono-1,5-lactones by ${ }^{1} \mathrm{H}$ NMR experiments involving NOESY measurements in solution.

\section{Results and Discussion}

2-O-Acyl-D-ribono-1,5-lactones 4 were prepared in high yield and gram amounts by acylation of $\mathbf{3}$ with acyl chlorides in excess pyridine followed by aqueous workup and crystallization (Scheme 2). The experimental procedure is straightforward and all reagents are easily available. It should be noted that application of alternative methods for acylation employing harmless reagents was severely restricted due to the poor solubility of $\mathbf{3}$ in many ordinary solvents.
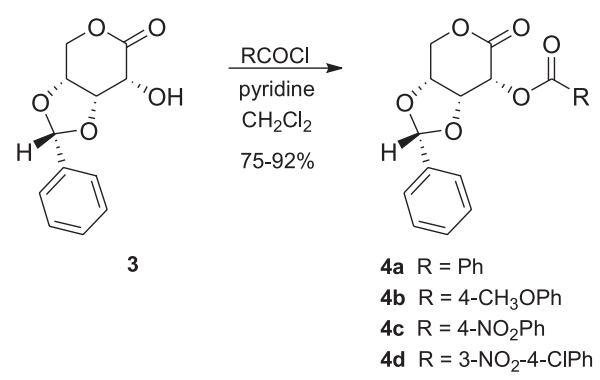

Scheme 2.
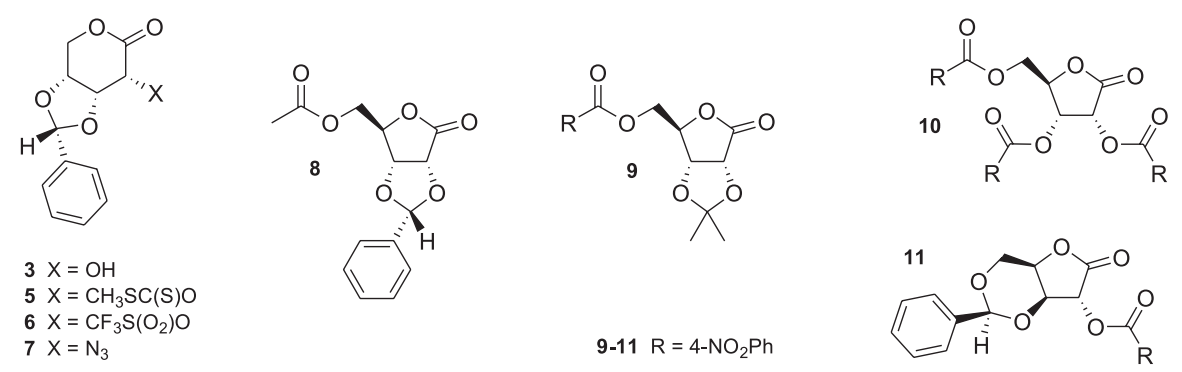

Scheme 3 .

The IR spectra of acylated compounds 4a-d (Table 1) showed the corresponding lactone carbonyl stretching at higher frequencies $\left(1756-1772 \mathrm{~cm}^{-1}\right)$ than those expected for typical six-membered lactones $\left(v_{\mathrm{C}=\mathrm{O}} 1735-1750 \mathrm{~cm}^{-1}\right) .{ }^{36}$ This observation is not entirely surprising because high frequencies have been previously reported for related $2-O$-substituted3,4-benzylidene-D-ribono-1,5-lactones such as (methylthio) thiocarbonyl (5) and trifluoromethanosulfonyl (6) derivatives (Scheme 3). ${ }^{14,24}$ Therefore, the observed carbonyl stretching in $1772 \mathrm{~cm}^{-1}$ for the nitro-substituted D-ribono-1,5-lactones $\mathbf{4 c , d}$ should not be considered as an indicative for the formation of a five- rather than a sixmembered lactone.

Unequivocal structural determination for lactone $\mathbf{4 c}$ was done by single crystal X-ray methods after a careful recrystallization of the solid product in acetone/ethanol. The pyranose ring adopts a boat-like conformation in the solid state, with the nitrobenzoyl group being positioned in a pseudo-equatorial orientation in order to minimize steric repulsions from the adjacent 3,4-dioxolanyl moiety (Figure 1). These results confirm that rearrangements coming from acyl migration, acetal isomerization or ring expansioncontraction did not operate under the stated reaction conditions for acylation of D-ribono-1,5-lactone 3. . $^{142,24}$

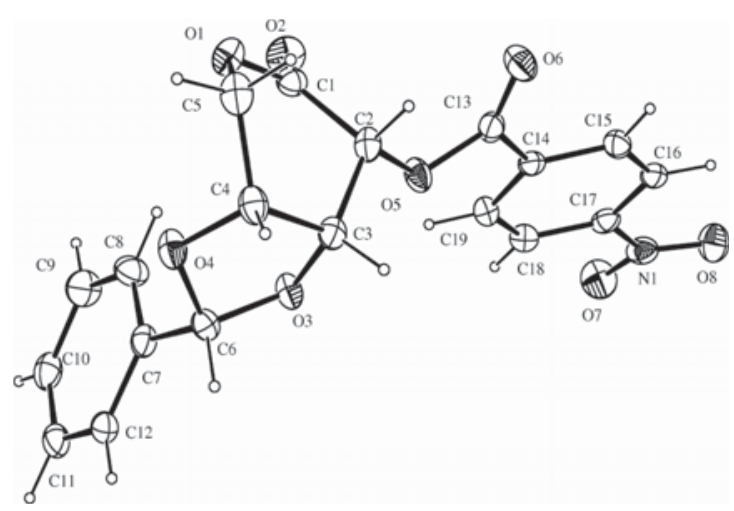

Figure 1. ORTEP ${ }^{43}$ view of D-ribono-1,5-lactone $4 \mathbf{c}\left(\mathrm{C}_{19} \mathrm{H}_{15} \mathrm{NO}_{8}\right)$, showing the atoms labeling and the $50 \%$ probability ellipsoids. The absolute configuration is assumed to be directly related to that of D-ribono-1,4lactone (1). 
Table 1. Infrared carbonyl stretching frequencies for 1,4- and 1,5-lactones

\begin{tabular}{llc}
\hline & $v_{\mathrm{C}=\mathrm{O}}$ Lactone $/ \mathrm{cm}^{-1}$ & Ref. \\
\hline $\mathbf{4 a}$ & 1760 & this work \\
$\mathbf{4 b}$ & 1756 & this work \\
$\mathbf{4 c}$ & 1772 & this work \\
$\mathbf{4 d}$ & 1772 & this work \\
$\mathbf{3}$ & 1750 & 21 \\
$\mathbf{5}$ & 1785 & 21 \\
$\mathbf{6}$ & 1770 & 13 \\
$\mathbf{7}$ & 1755 & 13 \\
$\mathbf{8}$ & 1760 & 14 \\
$\mathbf{9}$ & 1766 & 31 \\
$\mathbf{1 0}$ & 1810 & 31 \\
$\mathbf{1 1}$ & 1750 & 14 \\
\hline
\end{tabular}

Although the acylated D-ribono-1,5-lactone $\mathbf{4} \mathbf{c}$ was the only crystalline compound suitable for X-ray determination, a complete characterization of D-ribono-1,5-lactone $\mathbf{4}$ was accomplished by NMR spectroscopic data. Analysis by ${ }^{13} \mathrm{C}$ NMR spectroscopy has been previously proposed as a useful tool to establish the size of the lactone ring, supported by considerable differences in the chemical shifts found for C-3 and C-4 in a few lactones studied. ${ }^{13}$ However, a broader data compilation for 1,4- and 1,5-lactones revealed that no practical correlation could be rationalized (Table 2).

Table 2. Selected ${ }^{13} \mathrm{C}$ NMR chemical shifts $(\delta$, ppm) for $1,4-$ and 1,5-1actones ${ }^{\mathrm{a}}$

\begin{tabular}{lccccc}
\hline Lactone & C-2 & C-3 & C-4 & C-5 & Ref. \\
\hline $\mathbf{4 a}$ & 75.1 & 70.5 & 74.1 & 68.4 & this work \\
$\mathbf{4 b}$ & 75.2 & 70.2 & 74.1 & 68.3 & this work \\
$\mathbf{4 c}$ & 79.6 & 75.9 & 78.8 & 73.2 & this work \\
$\mathbf{3}$ & 67.7 & 73.3 & 76.7 & 66.8 & 21 \\
$\mathbf{5}^{\mathbf{b}}$ & 75.4 & 73.6 & 74.4 & 67.6 & 21 \\
$\mathbf{6}$ & 80.9 & 73.7 & 74.3 & 67.8 & 13 \\
$\mathbf{7}^{\mathbf{b}}$ & 58.9 & 73.1 & 76.3 & 67.3 & 13 \\
$\mathbf{1}$ & 69.4 & 68.7 & 85.5 & 60.5 & 37 \\
$\mathbf{8}^{\mathbf{b}}$ & $75.2^{\mathrm{c}}$ & $78.8^{\mathrm{c}}$ & $79.3^{\mathrm{c}}$ & 63.4 & 14 \\
$\mathbf{1 1}^{\mathbf{b}}$ & $74.8^{\mathrm{c}}$ & $73.4^{\mathrm{c}}$ & $74.6^{\mathrm{c}}$ & 65.0 & 14
\end{tabular}

${ }^{13} \mathrm{C} \mathrm{NMR}$ spectra $(100 \mathrm{MHz})$ were acquired using DMSO- $d_{6}$ as the solvent (unless otherwise specified), but no significant effect over the chemical shifts or coupling constants was observed when other common deuterated solvents such as $\mathrm{CDCl}_{3}$ and $\mathrm{C}_{6} \mathrm{D}_{6}$ were employed; ${ }^{\text {b}}$ Data obtained in $\mathrm{CDCl}_{3}$ as the solvent; ${ }^{\mathrm{c}}$ These assignments may have to be reversed.

After searching for general trends in the ${ }^{1} \mathrm{H}$ NMR to distinguish among several related sugar lactones, studies dealing with coupling constants $\left({ }^{3} J\right)$ revealed some useful patterns (Table 3). Small coupling between $\mathrm{H}-2$ and $\mathrm{H}-3$ were found for D-ribono-1,5-lactones 3-7 ( $\left.{ }^{3} J_{2,3} 1.5-3.4 \mathrm{~Hz}\right)$ in comparison with those for D-ribono-1,4-lactones 1,8-10 $\left({ }^{3} J_{2,3}=5.3-6.2 \mathrm{~Hz}\right)$. On the other hand, high values were encountered for $\mathrm{H}-3, \mathrm{H}-4$ coupling in D-ribono-1,5-lactones 3-7 $\left({ }^{3} J_{3,4} 7.9-8.2 \mathrm{~Hz}\right)$, while they are nearly absent in D-ribono-1,4-lactones 1,8-10 $\left({ }^{3} J_{3,4}<1 \mathrm{~Hz}\right)$. It is also worth noting that the very low values assigned for coupling between $\mathrm{H}-4$ and $\mathrm{H}-5 \mathrm{a} / \mathrm{b}$ in D-ribono-1,5-lactones 3-7 $\left({ }^{3} J_{4,5}<1.7 \mathrm{~Hz}\right)$, compared with those for D-ribono-1,4lactones 1,8-10 $\left({ }^{3} J_{4,5} 2.4-4.5 \mathrm{~Hz}\right)$, are probably associated with an adopted gauche conformation in solution with dihedral angles close to $60^{\circ}$, leading to weak spin-spin interactions as expected by the Karplus-Conroy equation. ${ }^{9,38}$

Table 3. Proton spin-spin coupling constants $\left({ }^{3} J_{n, m}, H z\right)$ for $1,4-$ and 1,5-lactones ${ }^{\mathrm{a}}$

\begin{tabular}{lllllc}
\hline Lactone & ${ }^{3} J_{2,3}$ & ${ }^{3} J_{3,4}$ & ${ }^{3} J_{4,5 \mathrm{a}}$ & ${ }^{3} J_{4,5 \mathrm{~b}}$ & Ref. \\
\hline $\mathbf{4 a}$ & 3.2 & 8.0 & $<1^{\mathrm{b}}$ & $<1^{\mathrm{b}}$ & this work \\
$\mathbf{4 b}$ & 3.4 & 8.0 & $<1^{\mathrm{b}}$ & $<1^{\mathrm{b}}$ & this work \\
$\mathbf{4 c}$ & 3.2 & 8.2 & $<1^{\mathrm{b}}$ & $<1^{\mathrm{b}}$ & this work \\
$\mathbf{4 d}$ & 1.5 & 8.2 & $<1^{\mathrm{b}}$ & $<1^{\mathrm{b}}$ & this work \\
$\mathbf{3}$ & 3.1 & 7.9 & 1.2 & $<1^{\mathrm{b}}$ & 21 \\
$\mathbf{5}^{\mathrm{d}}$ & 2.9 & 8.1 & $1.5^{\mathrm{c}}$ & $<1^{\mathrm{b}, \mathrm{c}}$ & 21 \\
$\mathbf{6}^{\mathrm{d}}$ & 3.4 & 8.0 & $1.7^{\mathrm{c}}$ & $<1^{\mathrm{b}, \mathrm{c}}$ & 13 \\
$\mathbf{7}^{\mathrm{d}}$ & 3.3 & 8.0 & $1.7^{\mathrm{c}}$ & $<1^{\mathrm{b}, \mathrm{c}}$ & 13 \\
$\mathbf{1}^{\mathrm{d}}$ & 5.3 & 0.8 & 3.6 & 3.6 & 37 \\
$\mathbf{8}^{\mathrm{d}}$ & 5.9 & $<1^{\mathrm{b}}$ & 2.4 & 2.8 & 14 \\
$\mathbf{9}^{\mathbf{e}}$ & 5.5 & $<1^{\mathrm{b}}$ & 3.0 & 3.0 & 31 \\
$\mathbf{1 0}^{\mathbf{1 0}}$ & 6.2 & $<1^{\mathrm{b}}$ & 4.5 & 4.5 & 31 \\
$\mathbf{1 1}^{\mathrm{d}}$ & $<1^{\mathrm{b}}$ & 2.7 & 2.3 & ${ }^{\mathrm{b}}$ & 14
\end{tabular}

${ }^{\mathrm{a}} \mathrm{H}$ NMR spectra $(400 \mathrm{MHz})$ were acquired using DMSO- $d_{6}$ as the solvent (unless otherwise specified), but no significant effect over the chemical shifts or coupling constants was observed when other common deuterated solvents such as $\mathrm{CDCl}_{3}$ and $\mathrm{C}_{6} \mathrm{D}_{6}$ were employed; ' ${ }^{\mathrm{b}}$ Coupling constants

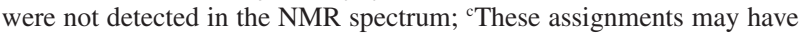
to be reversed; ${ }^{\mathrm{d}}$ Data obtained in $\mathrm{CDCl}_{3}$ as the solvent; ${ }^{\text {eData obtained }}$ in $\mathrm{C}_{6} \mathrm{D}_{6}$ as the solvent; ${ }^{\mathrm{f}}$ Values could not be assigned due to complex multiplicities found for $\mathrm{H}-4$ and $\mathrm{H}-5$.

The NOESY experiments performed with selected lactones not only confirmed the results deduced from coupling constant analysis but also revealed other important structural features (Table 4). Besides the anticipated presence of cross-peaks between $\mathrm{H}-3$ and $\mathrm{H}-4$ in the D-ribono-1,5-lactones 4a-c and 7 (H-3 and H-4 are syn), and its absence in D-ribono-1,4-lactones 9 and $\mathbf{1 0}(\mathrm{H}-3$ and $\mathrm{H}-4$ are anti), long-range contacts between $\mathrm{H}-2$ and $\mathrm{H}-5 \mathrm{~b}$ were also observed for all ribono-1,5-lactones but not for 1,4-lactones. Although no reasonable correlation was noted between $\mathrm{H}-4$ and $\mathrm{H}-5 \mathrm{a} / \mathrm{b}$ in D-ribono-1,5-lactones 4a-c and 7, this observation was expected based on the

Table 4. Important NOESY contacts for 1,4- and 1,5-1actones ${ }^{\mathrm{a}}$

\begin{tabular}{lcccc}
\hline Lactone & $\mathrm{H}-2, \mathrm{H}-3$ & $\mathrm{H}-2, \mathrm{H}-5 \mathrm{~b}$ & $\mathrm{H}-3, \mathrm{H}-4$ & $\mathrm{H}-4, \mathrm{H}-5 \mathrm{a} / \mathrm{b}$ \\
\hline $\mathbf{4 a}$ & $\mathrm{O}$ & $\mathrm{O}$ & $\mathrm{O}$ & $\mathrm{O}$ \\
$\mathbf{4 b}$ & $\mathrm{O}$ & $\mathrm{O}$ & $\mathrm{O}$ & $\mathrm{N}$ \\
$\mathbf{4 c}$ & $\mathrm{O}$ & $\mathrm{O}$ & $\mathrm{O}$ & $\mathrm{N}$ \\
$\mathbf{7}$ & $\mathrm{O}$ & $\mathrm{O}$ & $\mathrm{O}$ & $\mathrm{O}$ \\
$\mathbf{9}$ & $\mathrm{O}$ & $\mathrm{N}$ & $\mathrm{N}$ & $\mathrm{O}$ \\
$\mathbf{1 0}$ & $\mathrm{O}$ & $\mathrm{N}$ & $\mathrm{N}$ & $\mathrm{N}$ \\
\hline
\end{tabular}

Legend: O, Observed contact; N, Non-observed contact. 
almost non-existent ${ }^{3} J_{4,5 / b}$ values found for this class. The overall results clearly confirm that simple parameters such as coupling constants and NOE effects can be useful to support structural assignment for D-ribono-1,4- and D-ribono-1,5-lactones.

\section{Conclusions}

Structural assignments for $O$-benzylidene-D-ribono1,5-lactones were achieved using conventional NMR/ NOESY techniques and were confirmed by X-ray crystallography. In a general sense, the observed correlation between H-3 and H-4 is characteristic for D-ribono-1,5lactones, but not for D-ribono-1,4-lactones. In addition, significant spin-spin interaction between $\mathrm{H}-4$ and $\mathrm{H}-5$ are present in D-ribono-1,4-lactones, while they are absent in D-ribono-1,5-lactones. The particular trends featured by both D-ribono-1,4- and D-ribono-1,5-lactones can be helpful resources to account for their physicochemical characterization in a simple and reliable way.

\section{Experimental}

\section{General methods}

All chemicals were of reagent grade and were used as received. Melting points were determined using Microquímica MQPF301 apparatus and are uncorrected. Infrared spectra were acquired with Perkin-Elmer FTIR 1600 spectrometer using $\mathrm{KBr}$ for solid samples (range 4000-400 $\mathrm{cm}^{-1}$ ). ${ }^{1} \mathrm{H}$ NMR (400 MHz) and ${ }^{13} \mathrm{C}\left\{{ }^{1} \mathrm{H}\right\}$ NMR $(100 \mathrm{MHz})$ spectra were recorded with Varian 400 Mercury Plus spectrometer using DMSO- $d_{6}$ as solvent (unless otherwise noted) and $0.01 \%$ tetramethylsilane as internal reference in $5 \mathrm{~mm}$ o.d. sample tubes. For the ${ }^{1} \mathrm{H}$ spectra the conditions were as follows: deuterium internal lock, $\mathrm{T}=294 \mathrm{~K}$, pulse width 12.5 us, flip angle 90, acquisition time $4.1 \mathrm{~s}$, spectral width $6398 \mathrm{~Hz}$, delay time $1.0 \mathrm{~s}$, number of transients 32 and 34906 data points. ${ }^{13} \mathrm{C}$ spectra were recorded with pulse width 6.25 us, flip angle 45 , acquisition time $0.8 \mathrm{~s}$, spectral width $25125 \mathrm{~Hz}$, delay time $1.0 \mathrm{~s}$, number of transients 512 and 65326 data points. NOESY experiments were carried out with no degassed samples under the following conditions: spectral width $3597 \mathrm{~Hz}$, number of transients 16 , delay time $1.0 \mathrm{~s}$, number of increments 200, mixing time $0.8 \mathrm{~s}$ and 1024 data points at F1 dimension. Elemental analyses were conducted in a Carlo Erba CHNS EA-1110 equipment. Purifications by column chromatography were performed with silica gel (100-200 mesh particle size). $O$-Benzylidene-D-ribono1,5-lactone (3), ${ }^{21}$ azido derivative $7^{13}$ and D-ribono-1,4- lactones $\mathbf{9}, \mathbf{1 0}^{31}$ were readily obtained from D-ribono-1,4lactone (1) according to the described methods. NMR data for 5-O-(4'-nitrobenzoyl)-2,3-O-isopropylidene-Dribono-1,4-lactone (9) was aquired using perdeuterated benzene as the solvent: ${ }^{1} \mathrm{H}$ NMR $\left(400 \mathrm{MHz}, \mathrm{C}_{6} \mathrm{D}_{6}\right): \delta 1.36$ (s, 3H, $\left.\mathrm{CH}_{3}\right), 1.58\left(\mathrm{~s}, 3 \mathrm{H}, \mathrm{CH}_{3}\right), 3.89\left(\mathrm{dd}, 1 \mathrm{H}, J_{5 \mathrm{a}, 4} 3.0 \mathrm{~Hz}\right.$, $\left.J_{5 \mathrm{a}, 5 \mathrm{~b}} 12.5 \mathrm{~Hz}, \mathrm{H}-5 \mathrm{a}\right), 4.02$ (dd, $\left.1 \mathrm{H}, J_{5 \mathrm{~b}, 4} 3.0 \mathrm{~Hz}, \mathrm{H}-5 \mathrm{~b}\right), 4.14$ (d, $\left.1 \mathrm{H}, J_{3,2} 5.5 \mathrm{~Hz}, \mathrm{H}-3\right), 4.40$ (t, 1H, H-4), 4.71 (d, 1H, $\mathrm{H}-2)$, 7.40-7.85 (m, 7H, Ph, $\mathrm{H}_{\text {meta }}$ ), 8.15 (d, 2H, J $8.5 \mathrm{~Hz}$, $\mathrm{H}_{\text {orto }}$ ); its complete spectroscopic characterization was in agreement with published data. ${ }^{31}$

General procedure for the preparation of 2-O-acyl-Dribono-1,5-lactones $\mathbf{( 4 a - d )}$

To a solution containing $0.20 \mathrm{~g}(0.85 \mathrm{mmol})$ of Zinner's lactone (3) in $1.0 \mathrm{~mL}$ of anhydrous chloroform and 1.0 $\mathrm{mL}$ of dry pyridine under argon at $25{ }^{\circ} \mathrm{C}$ was added 1.3 mmol (1.5 equiv.) of the corresponding acyl chloride. After stirring for $15-18 \mathrm{~h}$, the reaction mixture was diluted with $\mathrm{CH}_{2} \mathrm{Cl}_{2}$ and successively washed with $5 \% \mathrm{HCl}, 5 \%$ $\mathrm{NaHCO}_{3}$, and $\mathrm{H}_{2} \mathrm{O}$. The organic extract was dried over $\mathrm{Na}_{2} \mathrm{SO}_{4}$, filtered, and concentrated under reduced pressure to give solid products that were purified by crystallization in acetone/ethanol (4a-c) or ethyl acetate $(\mathbf{4 d})$.

\section{2-O-Benzoyl-3,4-O-benzylidene-D-ribono-1,5-lactone} (4a)

92\% yield, white needles, mp $191-192{ }^{\circ} \mathrm{C}$; IR (KBr) $v_{\max } / \mathrm{cm}^{-1}: 1760,1740 ;{ }^{1} \mathrm{H}$ NMR: $\delta 4.51\left(\mathrm{~d}, 1 \mathrm{H}, J_{5 \mathrm{a}, 5 \mathrm{~b}}\right.$ $13.5 \mathrm{~Hz}, \mathrm{H}-5 \mathrm{a}), 4.74$ (d, 1H, H-5b), 4.82 (d, 1H, $J_{3,4} 8.0$ Hz, H-4), 5.02 (dd, 1H, J 3.3 Hz, H-3), 5.86 (s, 1H, H-6), $6.03(\mathrm{~d}, 1 \mathrm{H}, \mathrm{H}-2), 7.45$ (m, 5H, Ph), 7.56 (t, 2H, J 7.5 Hz, $\left.\mathrm{H}_{\text {meta }}\right), 7.72\left(\mathrm{t}, 1 \mathrm{H}, \mathrm{H}_{\text {para }}\right), 8.01\left(\mathrm{~d}, 2 \mathrm{H}, \mathrm{H}_{\text {orto }}\right) ;{ }^{13} \mathrm{C} \mathrm{NMR:}$ $\delta$ 68.4, 70.5, 74.1, 75.1, 103.8, 127.9 (2C), 129.1 (2C), 129.2, 129.7 (2C), 130.2 (2C), 130.7, 134.7, 136.4, 165.2, 167.5. Anal. Calc. for $\mathrm{C}_{19} \mathrm{H}_{16} \mathrm{O}_{6}: \mathrm{C}, 67.06 ; \mathrm{H}, 4.74$. Found: C, 66.77; H, 4.74.

\section{2-O-(4'-Methoxybenzoyl)-3,4-O-benzylidene-D-ribono- 1,5-lactone $\mathbf{( 4 b )}$}

$80 \%$ yield, white solid, mp $159-161{ }^{\circ} \mathrm{C}$; IR (KBr) $v_{\max } / \mathrm{cm}^{-1}: 1756,1726,1276 ;{ }^{1} \mathrm{H}$ NMR: $\delta 3.84(\mathrm{~s}, 3 \mathrm{H}$, $\left.\mathrm{OCH}_{3}\right), 4.50\left(\mathrm{~d}, 1 \mathrm{H}, J_{5 \mathrm{a}, 5 \mathrm{~b}} 13.5 \mathrm{~Hz}, \mathrm{H}-5 \mathrm{a}\right), 4.78$ (d, 1H, H-5b), 4.81 (d, 1H, $\left.J_{3,4} 8.0 \mathrm{~Hz}, \mathrm{H}-4\right), 5.00$ (dd, 1H, $J_{2,3}$ $3.4 \mathrm{~Hz}, \mathrm{H}-3), 5.87$ (s, 1H, H-6), 6.01 (d, 1H, H-2), 7.08 (d, 2H, J, 8.9 Hz, $\left.\mathrm{H}_{\text {meta }}\right), 7.45(\mathrm{~s}, 5 \mathrm{H}, \mathrm{Ph}), 7.96\left(\mathrm{~d}, 2 \mathrm{H}, \mathrm{H}_{\text {orto }}\right)$; ${ }^{13}$ C NMR: $\delta$ 56.3, 68.3, 70.2, 74.1, 75.2, 103.8, $114.9(2 \mathrm{C})$, 121.3, 127.9 (2C), 129.1 (2C), 130.7, 132.4 (2C), 136.4, 164.3, 164.8, 167.6. Anal. Calc. for $\mathrm{C}_{20} \mathrm{H}_{18} \mathrm{O}_{7}$ : C, 64.86; $\mathrm{H}$, 4.90. Found: C, 65.10; H, 4.92. 
2-O-(4'-Nitrobenzoyl)-3,4-O-benzylidene-D-ribono-1,5lactone $(4 \mathrm{c})$

$83 \%$ yield, yellow solid, mp $237-238^{\circ} \mathrm{C}$; IR (KBr) $v_{\max } / \mathrm{cm}^{-1}: 1772,1742,1528,1346 ;{ }^{1} \mathrm{H}$ NMR: $\delta 4.53(\mathrm{~d}$, $\left.1 \mathrm{H}, J_{5 \mathrm{a}, 5 \mathrm{~b}} 13.3 \mathrm{~Hz}, \mathrm{H}-5 \mathrm{a}\right), 4.77$ (d, 1H, H-5b), 4.84 (d, 1H, $\left.J_{3,4} 8.2 \mathrm{~Hz}, \mathrm{H}-4\right), 5.06$ (dd, $\left.1 \mathrm{H}, J_{2,3} 3.2 \mathrm{~Hz}, \mathrm{H}-3\right), 5.87$ (s, 1H, H-6), 6.11 (d, 1H, H-2), 7.44 (s, 5H, Ph), 8.24 (d, 2H, $\left.J 9.0 \mathrm{~Hz}, \mathrm{H}_{\text {orto }}\right), 8.38\left(\mathrm{~d}, 2 \mathrm{H}, \mathrm{H}_{\text {meta }}\right) ;{ }^{13} \mathrm{C} \mathrm{NMR}: \delta 73.2,75.9$, 78.8, 79.6, 108.6, 129.6 (2C), 132.7 (2C), 133.8 (2C), 135.5, 136.5 (2C), 139.2, 141.1, 156.0, 168.6, 172.0. Anal. Calc. for $\mathrm{C}_{19} \mathrm{H}_{15} \mathrm{NO}_{8}: \mathrm{C}, 59.22 ; \mathrm{H}, 3.92 ; \mathrm{N}, 3.64$. Found: C, $59.34 ; \mathrm{H}, 3.81 ; \mathrm{N}, 3.77$.

2-O-(4'-Chloro-3'-nitrobenzoyl)-3,4-O-benzylidene-Dribono-1,5-lactone $(\mathbf{4 d})$

$75 \%$ yield, yellow solid, mp $216-217{ }^{\circ} \mathrm{C}$; IR $(\mathrm{KBr})$ $v_{\max } / \mathrm{cm}^{-1}: 1772,1734,1540,1348 ;{ }^{1} \mathrm{H}$ NMR: $\delta 4.52(\mathrm{~d}$, $\left.1 \mathrm{H}, J_{5 \mathrm{a}, 5 \mathrm{~b}} 13.2 \mathrm{~Hz}, \mathrm{H}-5 \mathrm{a}\right), 4.77$ (d, 1H, H-5b), 4.83 (d, 1H, $\left.J_{3,4} 8.2 \mathrm{~Hz}, \mathrm{H}-4\right), 5.02$ (dd, $\left.1 \mathrm{H}, J_{2,3} 1.5 \mathrm{~Hz}, \mathrm{H}-3\right), 5.86$ (s, $1 \mathrm{H}, \mathrm{H}-6), 6.10$ (d, 1H, H-2), 7.44 (s, 5H, Ph), 7.96 (d, 1H, $\left.J 8.5 \mathrm{~Hz}, \mathrm{H}_{\text {meta }}\right), 8.23\left(\mathrm{~d}, 1 \mathrm{H}, \mathrm{H}_{\text {orto }}\right), 8.55\left(\mathrm{~s}, 1 \mathrm{H}, \mathrm{H}_{\text {orto }}\right)$. Anal. Calc. for $\mathrm{C}_{19} \mathrm{H}_{14} \mathrm{ClNO}_{8}$ : C, 54.36; H, 3.36; N, 3.34. Found: C, 54.40; H, 3.22; N, 3.25.

\section{Single-crystal X-ray structure determinations}

Low temperature X-ray diffraction data collection $(\varphi$ scans and $\omega$ scans with $\kappa$ offsets) of $\mathbf{4} \mathbf{c}$ was performed on an Enraf-Nonius Kappa-CCD diffractometer (95 mm CCD camera on $\kappa$-goniostat) using graphite-monochromated MoK $\alpha$ radiation $(0.71073 \AA$ ). Data were collected up to $50^{\circ}$ in $2 \theta$, with a redundancy of 4 . The final unit cell parameters were based on all reflections. Data collections were made using the COLLECT program, ${ }^{39}$ integration and scaling of the reflections were performed with the HKL Denzo-Scalepack system of programs. ${ }^{40}$ No absorption correction was applied. The structure was solved by direct methods with SHELXS-97.41 The model was refined by full-matrix least squares on $\mathrm{F}^{2}$ with SHELXL-97. ${ }^{42}$ All the hydrogen atoms were stereochemically positioned and refined with the riding model..$^{42}$ Hydrogen atoms were set isotropic with a thermal parameter $20 \%$ greater than the equivalent isotropic displacement parameter of the atom to which each one was bonded. The programs SHELXS-97, ${ }^{41}$ SHELXL- $97^{42}$ and ORTEP- $3^{43}$ were used within WinGX ${ }^{44}$ to prepare materials for publication.

\section{Supplementary Information}

Supplementary data are available free of charge at http://jbcs.sbq.org.br, as PDF file. Complete crystallographic data for the structural analysis of $\mathbf{4 c}$ have been deposited with the Cambridge Crystallographic Data Centre as supplementary publication number, CCDC 632862. Copies of available material may be obtained free of charge from the Director, Cambridge Crystallographic Data Centre, 12 Union Road, Cambridge, CB2 1EZ, UK. (fax: +44-1223-336033; e-mail: deposit@ccdc.cam.ac.uk; or via: www.ccdc.cam.ac.uk).

\section{Acknowledgments}

The authors wish to thank Central de Análises (Departamento de Química, UFSC, Florianópolis) for spectroscopic analysis. G. P. S. is grateful to CAPES and CNPq (Brazil) for a pre-doctoral fellowship. M. M. S. and J. E. thank CNPq for research fellowships. Financial support by MCT/CNPq (Brazilian Research Council), FAPESC (Santa Catarina State Research Council, Brazil), and PRONEX-2003 (CNPq/FAPESC) is also gratefully acknowledged. We also thank Dr. Jaroslav Zajicek and Dr. Anthony Serianni (Chemistry and Biochemistry Department, Notre Dame University) for their helpful suggestions.

\section{References}

1. Corma, A.; Iborra, S.; Velty, A.; Chem. Rev. 2007, 107, 2411.

2. Lichtenthaler, F. W.; Acc. Chem. Res. 2002, 35, 728.

3. Diéguez, M.; Pàmies, O.; Claver, C.; Chem. Rev. 2004, 104, 3189.

4. Gruner, S. A. W.; Locardi, E.; Lohof, E.; Kessler, H.; Chem. Rev. 2002, 102, 491.

5. Hanessian, S.; Lou, B.; Chem. Rev. 2000, 100, 4443.

6. Magaton, A. S.; Rubinger, M. M. M.; de Macedo Júnior, F. C.; Zambolim, L.; J. Braz. Chem. Soc. 2007, 18, 284.

7. de Oliveira, R. B.; Alves, R. J.; de Souza Filho, J. D.; Prado, M. A. F.; J. Braz. Chem. Soc. 2003, 14, 442.

8. Serianni, A. S. In Bioorganic Chemistry-Carbohydrates; Hecht, S. M., ed.; Oxford University Press: New York, 1999, pp. 244-312.

9. Liberek, B.; Tuwalska, D.; do Santos-Zounon, I.; Konitz, A.; Sikorski, A.; Smiatacz, Z.; Carbohydr. Res. 2006, 341, 2275.

10. Han, S.-Y.; Joullié, M. M.; Petasis, N. A.; Bigorra, J.; Corbera, J.; Font, J.; Ortuño, R. M.; Tetrahedron 1993, 49, 349.

11. Clode, D. M.; Can. J. Chem. 1977, 55, 4071.

12. Mastihubová, M.; Biely, P.; Carbohydr. Res. 2004, 339, 1353.

13. Baird, P. D.; Dho, J. C.; Fleet, G. W. J.; Peach, J. M.; Prout, K.; Smith, P. W.; J. Chem. Soc., Perkin 1 1987, 1785.

14. Han, S.-Y.; Joullié, M. M.; Fokin, V. V.; Petasis, N. A.; Tetrahedron: Asymmetry 1994, 5, 2535.

15. de Lederkremer, R. M.; Varela, O.; Adv. Carbohydr. Chem. Biochem. 1994, 50, 125. 
16. Bhat, K. L.; Chen, S.-Y.; Joullié, M. M.; Heterocycles 1985 , 23,691 .

17. Taylor, C. M.; Barker, W. D.; Weir, C. A.; Park, J. H.; J. Org. Chem. 2002, 67, 4466.

18. Burgos, E.; Roos, A. K.; Mowbray, S. L.; Salmon, L.; Tetrahedron Lett. 2005, 46, 3691.

19. Simone, M. I.; Soengas, R.; Newton, C. R.; Watkin, D. J.; Fleet, G. W. J.; Tetrahedron Lett. 2005, 46, 5761.

20. Zinner, H.; Voigh, H.; Voigh, J.; Carbohydr. Res. 1968, 7, 38.

21. Chen, S.-Y.; Joullié, M. M.; J. Org. Chem. 1984, 49, 2168.

22. Baggett, N.; Buchanan, J. G.; Fatah, M. Y.; Lachut, C. H.; McCullough, K. J.; Webber, J. M.; J. Chem. Soc., Chem. Commun. 1985, 1826.

23. Sanjayan, G. J.; Stewart, A.; Hachisu, S.; Gonzalez, R.; Watterson, M. P.; Fleet, G. W. J.; Tetrahedron Lett. 2003, 44, 5847.

24. Bruce, I.; Fleet, G. W. J.; Girdhar, A.; Haraldsson, M.; Peach, J. M.; Watkin, D. J.; Tetrahedron 1990, 46, 19.

25. Bigorra, J.; Font, J.; de Echagüen, C. O.; Ortuño, R. M.; Tetrahedron 1993, 49, 6717.

26. Ariza, J.; Font, J.; Ortuño, R. M.; Tetrahedron Lett. 1991, 32, 1979.

27. Takano, S.; Inomata, K.; Ogasawara, K.; Heterocycles 1988, $27,2413$.

28. Duus, J. Ø.; Gotfredsen, C. H.; Bock, K.; Chem. Rev. 2000, $100,4589$.

29. Freeman, F.; Robarge, K. D.; Carbohydr. Res. 1985, 137, 89.

30. Buchanan, J. G.; Edgar, A. R.; Rawson, D. I.; Shahidi, P.; Wightman, R. H.; Carbohydr. Res. 1982, 100, 75.

31. Sá, M. M.; Silveira, G. P.; Castilho, M. S.; Pavão, F.; Oliva, G.; Arkivoc 2002, 8, 112.
32. Leitão, A.; Andricopulo, A. D.; Oliva, G.; Pupo, M. T.; Marchi, A. A.; Vieira, P. C.; Silva, M. F. G. F.; Ferreira, V. F.; Souza, M. C. B. V.; Sá, M. M.; Moraes, V. R. S.; Montanari, C. A.; Bioorg. Med. Chem. Lett. 2004, 14, 2199.

33. Sá, M. M.; Meier, L.; Synlett 2006, 3474.

34. Mukaiyama, T.; Shiina, I.; Kobayashi, S.; Chem. Lett. 1990, 2201.

35. Hernández, R.; León, E. I.; Moreno, P.; Riesco-Fagundo, C.; Suárez, E.; J. Org. Chem. 2004, 69, 8437.

36. Silverstein, R. M.; Bassler, G. C.; Morril, T. C.; Espectrometric Identification of Organic Compounds, Livros Técnicos e Científicos: Rio de Janeiro, 1994, p. 98.

37. Horton, D.; Walaszek, Z.; Carbohydr. Res. 1982, 105, 111.

38. Breitmaier, E.; Structure Elucidation by NMR in Organic Chemistry: A Practical Guide, $3^{\text {rd }}$ ed., Wiley: England, 2002, pp. $42,43$.

39. Enraf-Nonius COLLECT; Nonius BV, Delft, The Netherlands, 1997-2000.

40. Otwinowski, Z.; Minor, W. In Methods in Enzymology; Carter Jr., C. W.; Sweet, R. M., eds.; Academic Press: New York, 1997; v. 276, pp. 307-326.

41. Sheldrick, M.; SHELXS-97. Program for Crystal Structure Resolution, Univ. of Göttingen: Göttingen, Germany, 1997.

42. Sheldrick, M.; SHELXL-97. Program for Crystal Structure Analysis, Univ. of Göttingen: Göttingen, Germany, 1997.

43. Farrugia, L. J.; J. Appl. Cryst. 1997, 30, 565.

44. Farrugia, L. J.; J. Appl. Cryst. 1999, 32, 837.

Received: May 30, 2007

Web Release Date: January 14, 2008

FAPESP helped in meeting the publication costs of this article. 
J. Braz Chem. Soc., Vol. 19, No. 1, S1-S3, 2008

\title{
Synthesis of Novel O-Acylated-D-ribono-1,5-lactones and Structural Assignment Supported by Conventional NOESY-NMR and X-ray Analysis
}

\author{
Marcus M. Sá, ${ }^{* a}{ }^{,}$Gustavo P. Silveira, ${ }^{a}$ Miguel S. B. Caro ${ }^{a}$ and Javier Ellena ${ }^{b}$ \\ ${ }^{a}$ Departamento de Química, Universidade Federal de Santa Catarina, 88040-900 Florianópolis-SC, Brazil \\ ${ }^{b}$ Instituto de Física de São Carlos, Universidade de São Paulo, CP 369, 13560-970 São Carlos-SP, Brazil
}

Table S1. Crystal data and structure refinement

\begin{tabular}{|c|c|}
\hline Empirical formula & $\mathrm{C}_{19} \mathrm{H}_{15} \mathrm{NO}_{8}$ \\
\hline Formula weight & 385.32 \\
\hline Temperature & $120(2) \mathrm{K}$ \\
\hline Wavelength & $0.71073 \AA$ \\
\hline Crystal system & Orthorhombic \\
\hline Space group & $\mathrm{P} 22_{1} 2_{1}$ \\
\hline Unit cell dimensions & $\begin{array}{l}a=6.7160(2) \AA \\
b=11.2840(3) \AA \\
c=22.2160(6) \AA\end{array}$ \\
\hline Volume & $1683.60(8) \AA^{3}$ \\
\hline $\mathrm{Z}$ & 4 \\
\hline Density (calculated) & $1.520 \mathrm{Mg} \mathrm{m}^{-3}$ \\
\hline Absorption coefficient & $0.121 \mathrm{~mm}^{-1}$ \\
\hline $\mathrm{F}(000)$ & 800 \\
\hline Crystal size & $0.24 \times 0.20 \times 0.16 \mathrm{~mm}^{3}$ \\
\hline Theta range for data collection & 2.57 to $25.00^{\circ}$ \\
\hline Index ranges & $-7 \leq h \leq 7,-11 \leq k \leq 11,-26 \leq l \leq 26$ \\
\hline Reflections collected & 2189 \\
\hline Independent reflections & $2189[\mathrm{R}(\mathrm{int})=0.0000]$ \\
\hline Completeness to theta $=25.00^{\circ}$ & $79.6 \%$ \\
\hline Refinement method & Full-matrix least-squares on $\mathrm{F}^{2}$ \\
\hline Computing $^{\mathrm{a}}$ & COLLECT, HKL Denzo and \\
\hline \multicolumn{2}{|l|}{ Scalepack, } \\
\hline Data / parameters & $2189 / 253$ \\
\hline Goodness-of-fit on $\mathrm{F}^{2}$ & 1.040 \\
\hline Final $R$ indices $[I>2 \sigma(I)]$ & $\mathrm{R} 1=0.0311, w \mathrm{R} 2=0.0747$ \\
\hline $\mathrm{R}$ indices (all data) & $\mathrm{R} 1=0.0345, w \mathrm{R} 2=0.0774$ \\
\hline Largest diff. peak and hole & 0.169 and -0.215 e. $\AA^{-3}$ \\
\hline
\end{tabular}

Table S2. Atomic coordinates $\left(\times 10^{4}\right)$ and equivalent isotropic displacement parameters $\left(\AA^{2} \times 10^{3}\right)$. U(eq) is defined as one third of the trace of the orthogonalized $\mathrm{U}_{\mathrm{ij}}$ tensor

\begin{tabular}{lcccc}
\hline & $\mathrm{x}$ & $\mathrm{y}$ & $\mathrm{z}$ & $\mathrm{U}(\mathrm{eq})$ \\
\hline $\mathrm{O}(1)$ & $5647(2)$ & $7162(1)$ & $2651(1)$ & $26(1)$ \\
$\mathrm{O}(2)$ & $4197(2)$ & $5609(2)$ & $2237(1)$ & $32(1)$ \\
$\mathrm{O}(3)$ & $9482(2)$ & $5128(1)$ & $2520(1)$ & $22(1)$ \\
$\mathrm{O}(4)$ & $9289(2)$ & $6547(1)$ & $3232(1)$ & $24(1)$ \\
$\mathrm{O}(5)$ & $7295(2)$ & $5105(1)$ & $1503(1)$ & $23(1)$ \\
$\mathrm{O}(6)$ & $6061(2)$ & $6088(1)$ & $704(1)$ & $29(1)$ \\
$\mathrm{O}(7)$ & $6025(3)$ & $-179(2)$ & $40(1)$ & $37(1)$ \\
$\mathrm{O}(8)$ & $6454(3)$ & $710(2)$ & $-814(1)$ & $36(1)$ \\
$\mathrm{N}(1)$ & $6279(3)$ & $715(2)$ & $-266(1)$ & $26(1)$ \\
$\mathrm{C}(1)$ & $5593(3)$ & $6267(2)$ & $2250(1)$ & $21(1)$ \\
$\mathrm{C}(2)$ & $7395(3)$ & $6185(2)$ & $1837(1)$ & $20(1)$ \\
$\mathrm{C}(3)$ & $9337(3)$ & $6213(2)$ & $2189(1)$ & $20(1)$ \\
$\mathrm{C}(4)$ & $9280(3)$ & $7195(2)$ & $2674(1)$ & $23(1)$ \\
$\mathrm{C}(5)$ & $7395(4)$ & $7917(2)$ & $2654(1)$ & $26(1)$ \\
$\mathrm{C}(6)$ & $10142(3)$ & $5432(2)$ & $3110(1)$ & $20(1)$ \\
$\mathrm{C}(7)$ & $9400(3)$ & $4503(2)$ & $3540(1)$ & $20(1)$ \\
$\mathrm{C}(8)$ & $7385(4)$ & $4212(2)$ & $3549(1)$ & $27(1)$ \\
$\mathrm{C}(9)$ & $6714(4)$ & $3309(2)$ & $3917(1)$ & $31(1)$ \\
$\mathrm{C}(10)$ & $8033(3)$ & $2681(2)$ & $4273(1)$ & $28(1)$ \\
$\mathrm{C}(11)$ & $10026(3)$ & $2981(2)$ & $4276(1)$ & $28(1)$ \\
$\mathrm{C}(12)$ & $10712(4)$ & $3890(2)$ & $3908(1)$ & $23(1)$ \\
$\mathrm{C}(13)$ & $6554(3)$ & $5179(2)$ & $938(1)$ & $20(1)$ \\
$\mathrm{C}(14)$ & $6490(3)$ & $3994(2)$ & $642(1)$ & $18(1)$ \\
$\mathrm{C}(15)$ & $6413(3)$ & $3972(2)$ & $13(1)$ & $21(1)$ \\
$\mathrm{C}(16)$ & $6370(3)$ & $2893(2)$ & $-288(1)$ & $21(1)$ \\
$\mathrm{C}(17)$ & $6343(3)$ & $1868(2)$ & $50(1)$ & $21(1)$ \\
$\mathrm{C}(18)$ & $6364(3)$ & $1870(2)$ & $673(1)$ & $23(1)$ \\
$\mathrm{C}(19)$ & $6468(3)$ & $2943(2)$ & $970(1)$ & $22(1)$ \\
\hline & & & &
\end{tabular}


Table S3. Full bond lengths $[\AA ̊]$ and angles $\left[{ }^{\circ}\right]$

\begin{tabular}{|c|c|c|c|}
\hline $\mathrm{O}(1)-\mathrm{C}(1)$ & $1.348(2)$ & $\mathrm{O}(2)-\mathrm{C}(1)-\mathrm{O}(1)$ & $120.18(18)$ \\
\hline $\mathrm{O}(1)-\mathrm{C}(5)$ & $1.450(3)$ & $\mathrm{O}(2)-\mathrm{C}(1)-\mathrm{C}(2)$ & $124.80(18)$ \\
\hline $\mathrm{O}(2)-\mathrm{C}(1)$ & $1.196(3)$ & $\mathrm{O}(1)-\mathrm{C}(1)-\mathrm{C}(2)$ & $115.01(18)$ \\
\hline $\mathrm{O}(3)-\mathrm{C}(6)$ & $1.426(2)$ & $\mathrm{O}(5)-\mathrm{C}(2)-\mathrm{C}(3)$ & $108.97(16)$ \\
\hline $\mathrm{O}(3)-\mathrm{C}(3)$ & $1.431(2)$ & $\mathrm{O}(5)-\mathrm{C}(2)-\mathrm{C}(1)$ & 109.11(17) \\
\hline $\mathrm{O}(4)-\mathrm{C}(6)$ & $1.409(3)$ & $C(3)-C(2)-C(1)$ & $111.68(13)$ \\
\hline $\mathrm{O}(4)-\mathrm{C}(4)$ & $1.438(2)$ & $\mathrm{O}(3)-\mathrm{C}(3)-\mathrm{C}(2)$ & $107.75(16)$ \\
\hline $\mathrm{O}(5)-\mathrm{C}(13)$ & $1.353(2)$ & $\mathrm{O}(3)-\mathrm{C}(3)-\mathrm{C}(4)$ & $104.90(12)$ \\
\hline $\mathrm{O}(5)-\mathrm{C}(2)$ & $1.428(2)$ & $\mathrm{C}(2)-\mathrm{C}(3)-\mathrm{C}(4)$ & $110.63(17)$ \\
\hline $\mathrm{O}(6)-\mathrm{C}(13)$ & $1.197(3)$ & $\mathrm{O}(4)-\mathrm{C}(4)-\mathrm{C}(5)$ & $107.74(15)$ \\
\hline $\mathrm{O}(7)-\mathrm{N}(1)$ & $1.228(3)$ & $\mathrm{O}(4)-\mathrm{C}(4)-\mathrm{C}(3)$ & $103.62(16)$ \\
\hline $\mathrm{O}(8)-\mathrm{N}(1)$ & $1.2234(19)$ & $\mathrm{C}(5)-\mathrm{C}(4)-\mathrm{C}(3)$ & $112.81(16)$ \\
\hline $\mathrm{N}(1)-\mathrm{C}(17)$ & $1.479(3)$ & $\mathrm{O}(1)-\mathrm{C}(5)-\mathrm{C}(4)$ & $111.25(17)$ \\
\hline $\mathrm{C}(1)-\mathrm{C}(2)$ & $1.522(3)$ & $\mathrm{O}(4)-\mathrm{C}(6)-\mathrm{O}(3)$ & $105.36(14)$ \\
\hline$C(2)-C(3)$ & $1.522(3)$ & $\mathrm{O}(4)-\mathrm{C}(6)-\mathrm{C}(7)$ & $111.47(15)$ \\
\hline$C(3)-C(4)$ & $1.546(3)$ & $\mathrm{O}(3)-\mathrm{C}(6)-\mathrm{C}(7)$ & $108.28(16)$ \\
\hline $\mathrm{C}(4)-\mathrm{C}(5)$ & $1.506(3)$ & $\mathrm{C}(12)-\mathrm{C}(7)-\mathrm{C}(8)$ & $119.40(19)$ \\
\hline $\mathrm{C}(6)-\mathrm{C}(7)$ & $1.503(3)$ & $C(12)-C(7)-C(6)$ & $120.8(2)$ \\
\hline $\mathrm{C}(7)-\mathrm{C}(12)$ & $1.387(3)$ & $\mathrm{C}(8)-\mathrm{C}(7)-\mathrm{C}(6)$ & $119.76(18)$ \\
\hline $\mathrm{C}(7)-\mathrm{C}(8)$ & $1.393(3)$ & $\mathrm{C}(9)-\mathrm{C}(8)-\mathrm{C}(7)$ & $120.0(2)$ \\
\hline $\mathrm{C}(8)-\mathrm{C}(9)$ & $1.382(3)$ & $\mathrm{C}(8)-\mathrm{C}(9)-\mathrm{C}(10)$ & $120.5(2)$ \\
\hline $\mathrm{C}(9)-\mathrm{C}(10)$ & $1.383(3)$ & $\mathrm{C}(11)-\mathrm{C}(10)-\mathrm{C}(9)$ & $119.9(2)$ \\
\hline $\mathrm{C}(10)-\mathrm{C}(11)$ & $1.381(3)$ & $C(10)-C(11)-C(12)$ & $119.9(2)$ \\
\hline $\mathrm{C}(11)-\mathrm{C}(12)$ & $1.390(3)$ & $C(7)-C(12)-C(11)$ & $120.3(2)$ \\
\hline $\mathrm{C}(13)-\mathrm{C}(14)$ & $1.491(3)$ & $\mathrm{O}(6)-\mathrm{C}(13)-\mathrm{O}(5)$ & $123.82(18)$ \\
\hline $\mathrm{C}(14)-\mathrm{C}(19)$ & $1.392(3)$ & $\mathrm{O}(6)-\mathrm{C}(13)-\mathrm{C}(14)$ & $124.70(15)$ \\
\hline$C(14)-C(15)$ & $1.397(2)$ & $\mathrm{O}(5)-\mathrm{C}(13)-\mathrm{C}(14)$ & $111.45(17)$ \\
\hline $\mathrm{C}(15)-\mathrm{C}(16)$ & $1.390(3)$ & $\mathrm{C}(19)-\mathrm{C}(14)-\mathrm{C}(15)$ & $120.52(18)$ \\
\hline$C(16)-C(17)$ & $1.379(3)$ & $C(19)-C(14)-C(13)$ & $122.19(14)$ \\
\hline $\mathrm{C}(17)-\mathrm{C}(18)$ & $1.385(2)$ & $C(15)-C(14)-C(13)$ & $117.28(18)$ \\
\hline C(18)-C(19) & $1.380(3)$ & $C(16)-C(15)-C(14)$ & $119.80(19)$ \\
\hline $\mathrm{C}(1)-\mathrm{O}(1)-\mathrm{C}(5)$ & $117.73(15)$ & $C(17)-C(16)-C(15)$ & $118.25(15)$ \\
\hline $\mathrm{C}(6)-\mathrm{O}(3)-\mathrm{C}(3)$ & $106.71(15)$ & $C(16)-C(17)-C(18)$ & $122.88(19)$ \\
\hline $\mathrm{C}(6)-\mathrm{O}(4)-\mathrm{C}(4)$ & $106.89(13)$ & $\mathrm{C}(16)-\mathrm{C}(17)-\mathrm{N}(1)$ & $118.69(14)$ \\
\hline $\mathrm{C}(13)-\mathrm{O}(5)-\mathrm{C}(2)$ & $116.52(15)$ & C(18)-C(17)-N(1) & $118.43(18)$ \\
\hline $\mathrm{O}(8)-\mathrm{N}(1)-\mathrm{O}(7)$ & $124.10(17)$ & $\mathrm{C}(19)-\mathrm{C}(18)-\mathrm{C}(17)$ & $118.65(18)$ \\
\hline $\mathrm{O}(8)-\mathrm{N}(1)-\mathrm{C}(17)$ & $118.25(17)$ & $C(18)-C(19)-C(14)$ & $119.85(15)$ \\
\hline $\mathrm{O}(7)-\mathrm{N}(1)-\mathrm{C}(17)$ & $117.64(14)$ & & \\
\hline
\end{tabular}


Table S4. Anisotropic displacement parameters $\left(\AA^{2} \times 10^{3}\right)$. The anisotropic displacement factor exponent takes the form: $-2 \pi^{2}\left[\mathrm{~h}^{2} a^{* 2} \mathrm{U}_{11}+.\right.$. $+2 \mathrm{~h} \mathrm{k} a^{*} b^{*} \mathrm{U}_{12}$

\begin{tabular}{|c|c|c|c|c|c|c|}
\hline & $\mathrm{U}_{11}$ & $\mathrm{U}_{22}$ & $\mathrm{U}_{33}$ & $\mathrm{U}_{23}$ & $\mathrm{U}_{13}$ & $\mathrm{U}_{12}$ \\
\hline $\mathrm{O}(1)$ & $28(1)$ & $23(1)$ & $26(1)$ & $-3(1)$ & $6(1)$ & $0(1)$ \\
\hline $\mathrm{O}(2)$ & $27(1)$ & $30(1)$ & $39(1)$ & 1(1) & $3(1)$ & $-7(1)$ \\
\hline $\mathrm{O}(3)$ & $30(1)$ & $18(1)$ & $16(1)$ & 1(1) & $-2(1)$ & $-2(1)$ \\
\hline $\mathrm{O}(4)$ & $39(1)$ & $16(1)$ & $18(1)$ & $0(1)$ & $0(1)$ & 1(1) \\
\hline $\mathrm{O}(5)$ & $33(1)$ & $20(1)$ & $16(1)$ & $-3(1)$ & $-4(1)$ & 2(1) \\
\hline $\mathrm{O}(6)$ & $40(1)$ & $23(1)$ & $23(1)$ & $0(1)$ & $-7(1)$ & $4(1)$ \\
\hline $\mathrm{O}(7)$ & $51(1)$ & $21(1)$ & $40(1)$ & $-2(1)$ & $-1(1)$ & 1(1) \\
\hline $\mathrm{O}(8)$ & $44(1)$ & $37(1)$ & $26(1)$ & $-12(1)$ & $-2(1)$ & $3(1)$ \\
\hline $\mathrm{N}(1)$ & $22(1)$ & $27(1)$ & $29(1)$ & $-7(1)$ & $-4(1)$ & $3(1)$ \\
\hline$C(1)$ & $22(1)$ & $18(1)$ & $22(1)$ & $3(1)$ & $-1(1)$ & $2(1)$ \\
\hline$C(2)$ & $28(1)$ & $14(1)$ & $17(1)$ & $-1(1)$ & 1(1) & $-1(1)$ \\
\hline$C(3)$ & $24(1)$ & $16(1)$ & $19(1)$ & $2(1)$ & $3(1)$ & 1(1) \\
\hline $\mathrm{C}(4)$ & $29(1)$ & $21(1)$ & $18(1)$ & 2(1) & 1(1) & $-4(1)$ \\
\hline$C(5)$ & $35(1)$ & $19(1)$ & $23(1)$ & $-1(1)$ & 1(1) & $-5(1)$ \\
\hline$C(6)$ & $20(1)$ & $22(1)$ & $18(1)$ & $0(1)$ & $-2(1)$ & $-1(1)$ \\
\hline$C(7)$ & $25(1)$ & $18(1)$ & $17(1)$ & $-1(1)$ & $2(1)$ & 1(1) \\
\hline $\mathrm{C}(8)$ & $27(1)$ & $26(1)$ & $28(1)$ & $8(1)$ & $-2(1)$ & $3(1)$ \\
\hline $\mathrm{C}(9)$ & $30(1)$ & $31(1)$ & $33(1)$ & $6(1)$ & $3(1)$ & $-3(1)$ \\
\hline $\mathrm{C}(10)$ & $40(2)$ & $23(1)$ & $22(1)$ & 2(1) & $7(1)$ & $2(1)$ \\
\hline $\mathrm{C}(11)$ & $39(2)$ & $26(1)$ & $18(1)$ & 1(1) & $-1(1)$ & $12(1)$ \\
\hline $\mathrm{C}(12)$ & $26(1)$ & $25(1)$ & $19(1)$ & $-3(1)$ & $-1(1)$ & $5(1)$ \\
\hline $\mathrm{C}(13)$ & $18(1)$ & $25(1)$ & $18(1)$ & $0(1)$ & 1(1) & $0(1)$ \\
\hline $\mathrm{C}(14)$ & $15(1)$ & $22(1)$ & $19(1)$ & $-2(1)$ & $-1(1)$ & $3(1)$ \\
\hline$C(15)$ & $17(1)$ & $26(1)$ & $20(1)$ & $1(1)$ & $-1(1)$ & $-1(1)$ \\
\hline$C(16)$ & $18(1)$ & $28(1)$ & $19(1)$ & $-2(1)$ & $-2(1)$ & $4(1)$ \\
\hline $\mathrm{C}(17)$ & $13(1)$ & $24(1)$ & $25(1)$ & $-5(1)$ & $-2(1)$ & 1(1) \\
\hline $\mathrm{C}(18)$ & $22(1)$ & $23(1)$ & $25(1)$ & 1(1) & $-1(1)$ & 2(1) \\
\hline C(19) & $23(1)$ & $26(1)$ & $17(1)$ & $0(1)$ & $0(1)$ & $3(1)$ \\
\hline
\end{tabular}

Table S6. Complete list of torsion angles $\left[{ }^{\circ}\right]$

$\mathrm{C}(5)-\mathrm{O}(1)-\mathrm{C}(1)-\mathrm{O}(2)$

$\mathrm{C}(5)-\mathrm{O}(1)-\mathrm{C}(1)-\mathrm{C}(2)$

$\mathrm{C}(13)-\mathrm{O}(5)-\mathrm{C}(2)-\mathrm{C}(3)$

$\mathrm{C}(13)-\mathrm{O}(5)-\mathrm{C}(2)-\mathrm{C}(1)$

$\mathrm{O}(2)-\mathrm{C}(1)-\mathrm{C}(2)-\mathrm{O}(5)$

$\mathrm{O}(1)-\mathrm{C}(1)-\mathrm{C}(2)-\mathrm{O}(5)$

$\mathrm{O}(2)-\mathrm{C}(1)-\mathrm{C}(2)-\mathrm{C}(3)$

$\mathrm{O}(1)-\mathrm{C}(1)-\mathrm{C}(2)-\mathrm{C}(3)$

$\mathrm{C}(6)-\mathrm{O}(3)-\mathrm{C}(3)-\mathrm{C}(2)$

$\mathrm{C}(6)-\mathrm{O}(3)-\mathrm{C}(3)-\mathrm{C}(4)$

$\mathrm{O}(5)-\mathrm{C}(2)-\mathrm{C}(3)-\mathrm{O}(3)$

$\mathrm{C}(1)-\mathrm{C}(2)-\mathrm{C}(3)-\mathrm{O}(3)$

$\mathrm{O}(5)-\mathrm{C}(2)-\mathrm{C}(3)-\mathrm{C}(4)$

$\mathrm{C}(1)-\mathrm{C}(2)-\mathrm{C}(3)-\mathrm{C}(4)$

$\mathrm{C}(6)-\mathrm{O}(4)-\mathrm{C}(4)-\mathrm{C}(5)$

$\mathrm{C}(6)-\mathrm{O}(4)-\mathrm{C}(4)-\mathrm{C}(3)$

$\mathrm{O}(3)-\mathrm{C}(3)-\mathrm{C}(4)-\mathrm{O}(4)$

$\mathrm{C}(2)-\mathrm{C}(3)-\mathrm{C}(4)-\mathrm{O}(4)$

$\mathrm{O}(3)-\mathrm{C}(3)-\mathrm{C}(4)-\mathrm{C}(5)$

$\mathrm{C}(2)-\mathrm{C}(3)-\mathrm{C}(4)-\mathrm{C}(5)$

$\mathrm{C}(1)-\mathrm{O}(1)-\mathrm{C}(5)-\mathrm{C}(4)$

$\mathrm{O}(4)-\mathrm{C}(4)-\mathrm{C}(5)-\mathrm{O}(1)$

$\mathrm{C}(3)-\mathrm{C}(4)-\mathrm{C}(5)-\mathrm{O}(1)$

$\mathrm{C}(4)-\mathrm{O}(4)-\mathrm{C}(6)-\mathrm{O}(3)$

$\mathrm{C}(4)-\mathrm{O}(4)-\mathrm{C}(6)-\mathrm{C}(7)$

$\mathrm{C}(3)-\mathrm{O}(3)-\mathrm{C}(6)-\mathrm{O}(4)$

$\mathrm{C}(3)-\mathrm{O}(3)-\mathrm{C}(6)-\mathrm{C}(7)$

$\mathrm{O}(4)-\mathrm{C}(6)-\mathrm{C}(7)-\mathrm{C}(12)$

$\mathrm{O}(3)-\mathrm{C}(6)-\mathrm{C}(7)-\mathrm{C}(12)$

$\mathrm{O}(4)-\mathrm{C}(6)-\mathrm{C}(7)-\mathrm{C}(8)$

$\mathrm{O}(3)-\mathrm{C}(6)-\mathrm{C}(7)-\mathrm{C}(8)$

$\mathrm{C}(12)-\mathrm{C}(7)-\mathrm{C}(8)-\mathrm{C}(9)$

$\mathrm{C}(6)-\mathrm{C}(7)-\mathrm{C}(8)-\mathrm{C}(9)$

$\mathrm{C}(7)-\mathrm{C}(8)-\mathrm{C}(9)-\mathrm{C}(10)$

C(8)-C(9)-C(10)-C(11)

$\mathrm{C}(9)-\mathrm{C}(10)-\mathrm{C}(11)-\mathrm{C}(12)$

$\mathrm{C}(8)-\mathrm{C}(7)-\mathrm{C}(12)-\mathrm{C}(11)$

$\mathrm{C}(6)-\mathrm{C}(7)-\mathrm{C}(12)-\mathrm{C}(11)$

$\mathrm{C}(10)-\mathrm{C}(11)-\mathrm{C}(12)-\mathrm{C}(7)$

$\mathrm{C}(2)-\mathrm{O}(5)-\mathrm{C}(13)-\mathrm{O}(6)$

$\mathrm{C}(2)-\mathrm{O}(5)-\mathrm{C}(13)-\mathrm{C}(14)$

$\mathrm{O}(6)-\mathrm{C}(13)-\mathrm{C}(14)-\mathrm{C}(19)$

$\mathrm{O}(5)-\mathrm{C}(13)-\mathrm{C}(14)-\mathrm{C}(19)$

$\mathrm{O}(6)-\mathrm{C}(13)-\mathrm{C}(14)-\mathrm{C}(15)$

$\mathrm{O}(5)-\mathrm{C}(13)-\mathrm{C}(14)-\mathrm{C}(15)$

$\mathrm{C}(19)-\mathrm{C}(14)-\mathrm{C}(15)-\mathrm{C}(16)$

C(13)-C(14)-C(15)-C(16)

$\mathrm{C}(14)-\mathrm{C}(15)-\mathrm{C}(16)-\mathrm{C}(17)$

$\mathrm{C}(15)-\mathrm{C}(16)-\mathrm{C}(17)-\mathrm{C}(18)$

$\mathrm{C}(15)-\mathrm{C}(16)-\mathrm{C}(17)-\mathrm{N}(1)$

$\mathrm{O}(8)-\mathrm{N}(1)-\mathrm{C}(17)-\mathrm{C}(16)$

$\mathrm{O}(7)-\mathrm{N}(1)-\mathrm{C}(17)-\mathrm{C}(16)$

$\mathrm{O}(8)-\mathrm{N}(1)-\mathrm{C}(17)-\mathrm{C}(18)$

$\mathrm{O}(7)-\mathrm{N}(1)-\mathrm{C}(17)-\mathrm{C}(18)$

$\mathrm{C}(16)-\mathrm{C}(17)-\mathrm{C}(18)-\mathrm{C}(19)$

$\mathrm{N}(1)-\mathrm{C}(17)-\mathrm{C}(18)-\mathrm{C}(19)$

$\mathrm{C}(17)-\mathrm{C}(18)-\mathrm{C}(19)-\mathrm{C}(14)$

$\mathrm{C}(15)-\mathrm{C}(14)-\mathrm{C}(19)-\mathrm{C}(18)$

C(13)-C(14)-C(19)-C(18)

$178.85(17) \quad 53$

$-1.3(2) \quad 54$

$-139.83(16)$

$\begin{array}{ll}97.99(18) & 56\end{array}$

171.06(15) 57

$-129.6(2) \quad 58$

$50.5(2)$

$-136.10(16)$

$-18.2(2)$

$-51.57(18)$

69.04(19)

$-165.71(14)$

$-45.1(2)$

143.29(17)

23.5(2)

$-3.1(2)$

112.88(17)

$-119.29(17)$

$-3.4(2)$

$-49.87(19)$

$-63.09(18)$

50.66(19)

$-35.8(2)$

$-153.08(17)$

$33.6(2)$

152.96(16)

$-120.3(2)$

124.27(19)

$62.8(2)$

$-52.6(2)$

$-0.9(3)$

176.02(19)

$-0.5(3)$

1.9(3)

$-1.9(3)$

1.0(3)

$-175.95(18)$

$0.4(3)$

2.8(3)

-178.92(17)

$-159.5(2)$

22.2(3)

19.3(3)

$-158.93(18)$

$-1.8(3)$

179.40(19)

2.0(3)

$-0.2(3)$

179.44(18)

$7.2(3)$

$-171.8(2)$

$-173.08(18)$

7.9(3)

$-1.7(3)$

178.59(18)

1.9(3)

$-0.2(3)$

178.6(2) 